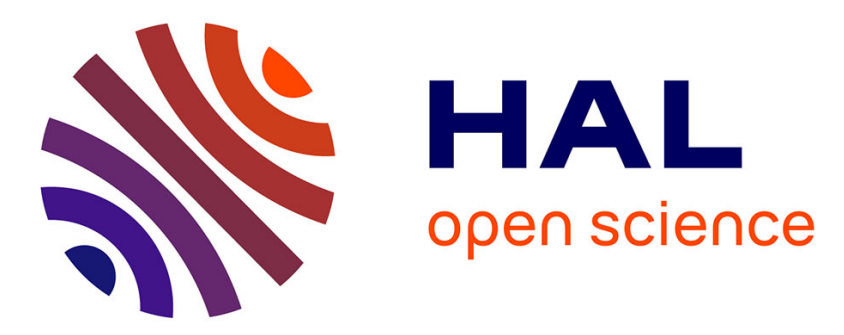

\title{
ORIGIN OF PARITY-NON-CONSERVATION IN NEUTRON-NUCLEUS INTERACTIONS
}

B. Desplanques

\section{To cite this version:}

B. Desplanques. ORIGIN OF PARITY-NON-CONSERVATION IN NEUTRON-NUCLEUS INTERACTIONS. Workshop on Reactor Based Fundamental Physics, 1983, Grenoble, France. pp.C3-55-C370, 10.1051/jphyscol:1984312 . jpa-00224026

\section{HAL Id: jpa-00224026 https://hal.science/jpa-00224026}

Submitted on 1 Jan 1984

HAL is a multi-disciplinary open access archive for the deposit and dissemination of scientific research documents, whether they are published or not. The documents may come from teaching and research institutions in France or abroad, or from public or private research centers.
L'archive ouverte pluridisciplinaire HAL, est destinée au dépôt et à la diffusion de documents scientifiques de niveau recherche, publiés ou non, émanant des établissements d'enseignement et de recherche français ou étrangers, des laboratoires publics ou privés. 
JOURNAL DE PHYSIQUE

Colloque C3, supplément au n³, Tome 45, mars 1984

page $\mathrm{C} 3-55$

\title{
ORIGIN OF PARITY-NON-CONSERVATION IN NEUTRON-NUCLEUS INTERACTIONS
}

\author{
B. Desplanques ${ }^{\star}$
}

Institut Laue-Langevin, 156 x, 38042 Grenoble Cedex, France

\begin{abstract}
Résumé - Les différentes êtapes qui mènent de la non-conservation de la paríté entre quarks à la non-conservation de la parité dans les noyaux sont passées en revue. Les prédictions concernant différentes observables ne conservant pas la parité sont comparées aux mesures et il est montré qu'assez peu est connu sur les interactions faibles impliquant des neutrons. L'intêrêt de certaines expériences mettant en jeu ces neutrons est discuté.
\end{abstract}

Abstract - The different steps which lead from parity-non-conservation at the quark level to parity-non-conservation in nuclear systems are reviewed. Current predictions for various parity-non-conserving observables are compared to measurements and it is shown that little is known about the parity non-conserving neutron-nucleus interaction. The interest of a few measurements which may provide some information about this interaction is discussed.

The origin of parity-non-conservation in nuclear processes is generally attributed to weak interactions which are now assumed to be well described by the GlashowWeinberg-Salam mode1, as far as the quark sector involved here is concerned /1/. Although there is no serious reason to doubt about the validity of this model in the present domain, the link between theory and experiment is certainly weaker here than in other domains. We are indeed dealing with a piece of the electroweak interaction whose effects are masked by the strong interaction. As a consequence, they are very difficult to observe since their average magnitude at low energy is of the order of $10^{-6}-10^{-7}$. Experimentalists have therefore tried in past years to look at cases where some enhancement was expected and, in one case, an effect as large as $7 \%$ has been observed. The difficulty to measure effects with the current expected magnitude has not however stopped their efforts. In this respect, results now obtained by several groups in proton-proton scattering around $15 \mathrm{MeV}$ or $50 \mathrm{MeV}$, roughly in agreement with each other, are worthwhile to be mentioned. Difficulties are also present on the theoretical side. Describing parity-non-conserving (pnc) effects in a system involving several nucleons from an elementary interaction given in terms of quarks is an enormous task. As also evidenced by the strong interaction, where much more information is available, such calculations involve ingredients which are not always under control. Nevertheless, it appears that reasonable assumptions account for the magnitude of effects observed in quite different processes. In some cases, the agreement between theory and experiment is particularly good and pertinent features are reproduced. In other cases, estimates are significantly larger or lower than present measurements, thus indicating that there are still theoretical or experimental deficiencies in dealing with these effects.

An important ingredient in calculating pnc effects in complex nuclei is the pnc nucleon-nucleon (NN) interaction which is assumed to result from the exchange of mesons, as for the strong NN interaction. In this picture, parity-non-conservation is 
introduced at the level. of the meson-nucleon interaction and it is there that pnc interactions between quarks are involved. The different assumptions which allow to determine this pnc NN interaction from the pnc quark-quark interaction are discussed in the first section together with the corresponding uncertainties. Section 2 is devoted to the calculation of observables from the pnc NN interaction. It concerns short-range correlations in the NN system, which leads to the introduction of 5 elementary transition amplitudes. These amplitudes may reveal useful to analyze the results independently of the detailed nature of the processes which produce them. It also concerns nuclear structure considerations, leading in particular to some enhancement of pnc effects. In section 3, we show that present theoretical calculations give a good account of several observables involving a "polarized" proton (in pp scattering as well as in several odd-proton nuclei). The limits of the agreement as well as the absence of measurements providing information on the part of the pnc NN interaction involving directly a "polarized" neutron are emphasized. We finally discuss in section 4 several processes involving neutrons and their respective interest with regard to parity-non-conservation in the NN system.

As indicated by the title, the present review is essentially devoted to pnc neutronnucleus interactions. While it contains a large part dealing with general concepts about pnc nuclear forces, it does not pretend to give a complete review of the work recently done on them. Only pieces of this work, selected for their interest with respect to our purpose, are intented to be mentioned.

I - PARITY-NON-CONSERVATION : FROM QUARKS TO NUCLEONS

a) the-_pnc_.NN inte-

Our current views about the pnc NN interaction are largely suggested by the ones we have for the strong NN interaction which is usually assumed to result from the exchange of mesons. In this interaction, the one-pion-exchange is responsible for the long range part. The two-pion exchange in a relative S state(" $\sigma$ ") explains a large part of the attraction at intermediate distances $(\simeq 1.5 \mathrm{fm})$, whereas in a $p$ state $(\rho)$, it plays an important role for some correlations. The exchange of three pions, with the quantum numbers of the w-particle is essential to explain the short-range repulsion between nucleons. As they explain the gross features of the strong $\mathrm{NN}$ interaction, it is reasonable to expect that they will do similarly for the pne part of the NN interaction. There are however striking differences due to the nature of the weak interaction. On one hand, isospin is not conserved, so that there are contributions from $\Delta T=0,1$ and 2 parts. On the other hand, the assumption that PC is conserved, which holds to a good approximation, implies that the exchange of any neutral system of mesons with a zero total angu1 ar momentum is forbidden (extension of the Barton's theorem $/ 2 /$ ). Thus, the exchange of the pseudoscalar particles $\pi^{\circ}$ and $\eta^{\circ}$ are excluded, as we 11 as the exchange of $2 \pi$ in a relative S state, whose role in binding nucleons together is well known. The long-range contribution is therefore due to the exchange of a charged pion. This contribution has played and is still playing a particular role. It depends only on the isovector part of the weak interaction $(\Delta T=1)$, which itself is dominated by the contribution of the neutral current part. Furthermore, its contribution in nuclear systems is less sensitive to short range correlations and can be calculated more reliably than any other contribution. There are shorter range contributions due to $\rho(2 \pi$ in a $P$ state) or $\omega$ exchanges. In contrast to the $\pi$ exchange, their number is not seriously limited by the conservation of PC.

The resulting pnc NN interactions is represented on Fig. 1. The various: exchanges involve both the strong and weak (pnc) interactions of mesons with nucleons. The first one, represented by a circle (0), are known, whereas the second ones, which are represented by a square (a) and contain all the information involving paritynon-conservation, have to be determined. Independently of their actual value, and without restriction, they may be parametrized /3/ with $f_{\pi}$ for the $\pi N N$ interaction, $h_{\rho}^{0}, h_{\rho}^{1}, h_{\rho}^{2}$ for the vector $\rho$ NN couplings corresponding to $\Delta T=0,1$ and $2, h_{\rho}^{l}$ for the tensor $\rho$ NN coupling with $\Delta \mathrm{T}=1$ and $h_{\omega}^{0}$, $h_{\omega}^{1}$ for the vector $\omega N N$ couplings with 
$\Delta \mathrm{T}=0$ and 1.
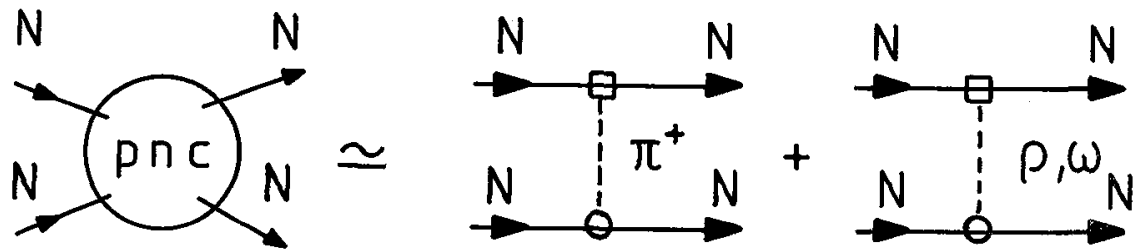

Fig. 1 - Diagramatic representation of the pnc NN interaction.

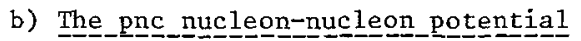

Using the non-relativistic limit, the pnc NN potential resulting from the various meson exchanges discussed above can be written :

$$
\begin{aligned}
& v_{12}^{P N C}=i \frac{1}{2}\left(\vec{\tau}_{1} \times \vec{\tau}_{2}\right)^{z}\left(\vec{\sigma}_{1}+\vec{\sigma}_{2}\right)\left[\frac{\vec{p}}{\bar{M}}, \frac{g_{\pi N N} f_{\pi}}{\sqrt{2}} f_{\pi}(r)-g_{\rho} h_{\rho}{ }^{\prime} f_{\rho}(r)\right] \\
& -g_{\rho}\left(h_{\rho}^{0} \vec{\tau}_{1} \cdot \vec{\tau}_{2}+\frac{1}{2} h_{\rho}^{1}\left(\tau_{1}^{z}+\tau_{2}^{z}\right)+\frac{1}{2} \frac{h_{\rho}^{2}}{\sqrt{6}}\left(3 \tau_{1}^{z} \tau_{2}^{z}-\vec{\tau}_{1} \cdot \vec{\tau}_{2}\right)\right) \\
& x\left\{\left(\vec{\sigma}_{1}-\vec{\sigma}_{2}\right)\left\{\frac{\vec{P}}{\bar{M}}, f_{\rho}(r)\right\}+i\left(1+x_{v}\right)\left(\vec{\sigma}_{1} x \vec{\sigma}_{2}\right)\left[\frac{\vec{P}}{M}, f_{\rho}(r)\right]\right\} \\
& \left.-g_{\omega}\left(h_{\omega}^{0}+\frac{1}{2} h_{\omega}^{l}\left(\tau_{1}^{z}+\tau_{2}^{z}\right)\right) \times\left(\vec{\sigma}_{1}-\vec{\sigma}_{2}\right)\left\{\frac{\vec{P}}{M}, f_{\omega}(x)\right\}+i\left(1+x_{s}\right)\left(\vec{\sigma}_{1} \times \vec{\sigma}_{2}\right)\left[\frac{\vec{P}}{M}, f_{\omega}(r)\right]\right) \\
& -\left(g_{\omega} h_{\omega}^{1}-g_{\rho} h_{\rho}^{1}\right) \frac{1}{2}\left(\tau_{1}^{z}-\tau_{2}^{2}\right)\left(\vec{\sigma}_{1}+\vec{\sigma}_{2}\right)\left\{\frac{\vec{p}}{M}, f_{\rho, \omega}(r)\right\} \\
& \text { where } \vec{p}=\frac{1}{2}\left(\vec{p}_{1}-\vec{p}_{2}\right), f_{\pi}(r)=\frac{e^{-m_{\pi} r}}{4 \pi r}, f_{\rho}(r)=f_{\omega}(r)=\frac{e^{-m_{\rho} r}}{4 \pi r}
\end{aligned}
$$

While signs of strong and weak meson-nucleon coupling constants depend on phase conventions, the sign of their product is quite relevant. Throughout this paper our signs will refer to the above potential where the strong coupling constants are assumed to be positive whereas their magnitude is given by :

$$
\frac{g_{\pi N N}^{2}}{4 \pi}=14.4 \frac{g_{\rho N N}^{2}}{4 \pi}=\frac{1}{9} \cdot \frac{g_{\omega N N}^{2}}{4 \pi}=0.62
$$

The tensor $\rho N N$ and wNN couplings which appear in (1), $x_{\mathrm{y}}$ and $x_{s}$, will be given the values of the isovector and isoscalar nucleon magnetic moments $\mu_{v}$ and $\mu_{s}$. This choice may be open to discussion in the case of $x_{v}$ which is known to be significantly larger than $\mu_{v} / 4 /$. This is one of the numerous uncertainties pertaining to the description of pnc effects.

\section{c) The_pnc_meson-nucleon_coup 1 ing congonstants}

The connection between parity-non-conservation in the NN system and in the weak interaction given in terms of quarks is generally made at the level of the mesonnucleon coupling constants. Although the original task is somewhat simplified, it is not solved as the calculation of these coupling constants requires models for describing nucleons and mesons in terms of quarks. The difficulties are very similar to those encountered in calculating non-leptonic hyperon decay amplitudes, but using experimental information on these amplitudes can alleviate the uncertainties appearing in the present case. The main contributions in terms of quarks are represented on Fig. 2. In the first diagram a), the meson is created from one quark line and its contribution can be factorized into a product of one body matrix elements involving separately the meson and the nucleon. Such a contribution, 
which has been known to be present in $\rho N N$ and wNN weak coupling constants since a long time, is also present in the $\pi N N$ weak coupling constant. This is due to the

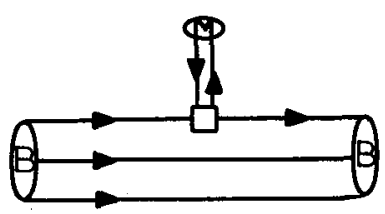

a)

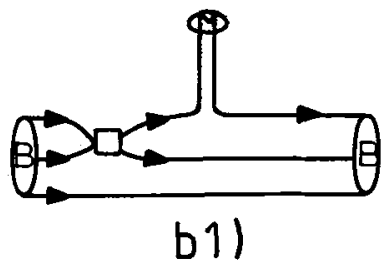

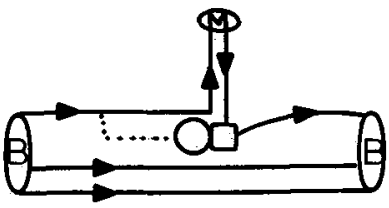

c)

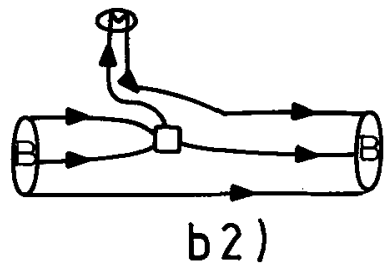

Fig. 2 - Various quark contributions to meson-nucleon coupling constants

fact that the Glashow-Weinberg-Salam model of electroweak interactions is not symmetrical in the exchange of vector and axial natures of currents. In diagrams b), the weak interaction is acting on two quarks of the same nucleon. Its contribution has a structure different from the previous one, since it involves a two-body matrix element and, to some extent, it can be interpreted as arising from parity-admixture into the nucleon. At this point, it is appropriate to comment about the separation we made between diagrams b1) and b2). The first one may correspond to the quark excitation inside the nucleon from an $s$ to a $p$ orbit, whereas the other one would involve the excitation of a quark-antiquark pair. Both contributions are present, but the relative weight can be quite different depending on the nature of the meson which is considered : pseudo-scalar ( $\pi$ ) or vector $(\rho, \omega)$. This weight is not essential in the case of the $\pi$ meson because PCAC and current algebra generally allow to sum up all the contributions. This is not the case however for the $\rho$ meson. The validity of the vector currentmeson field identity would imply that these different contributions cancel each other whereas the validity of the SU(6) $)_{\mathrm{W}}$ symmetry would imply that only the 2 nd contribution (b2) is present. Finally diagram c) represents a contribution due to the excitation of $q \bar{q}$ pairs in the nucleon. By an appropriate redefinition of the effective weak interaction, which already contains some effect due to gluon exchange, the pair contribution might be included in contribution of diagram a). It is considered here separately because of its possible sensitivity to SU(3) breaking effects. Indeed, the weight of a $s \bar{s}$ pair (strangeness carrying quarks) which is often involved here is likely to be smaller than the weight of the d $\bar{d}$ pair which is involved in nonleptonic hyperon decays.

Having described the different processes which occur at the quark leve1, it is now possible to provide estimates for the meson-nucleon coupling constants. As shown above, there is no unambiguous relationship between the quantities of interest here and nonleptonic hyperon decays. It results some uncertainty which is quite large as it can be seen from the first column of Table 1. Together with these extreme values, authors of these results /3/ have provided some "best values" corresponding to reasonable choices for the undetermined inputs. Although there is no strong theoretical reason to do it, we will mainly refer in the following to these particular values as they explain non-trivial pnc effects. In the best case, they just may be the right ones and it would remain to find a deeper justification to the different choices which have been made. In the worse case, they might appropriately simulate some unknown physics which should show up in other circumstances. 
Table I - Range and "best" estimate of Glashow-Weinberg-Salam parameters

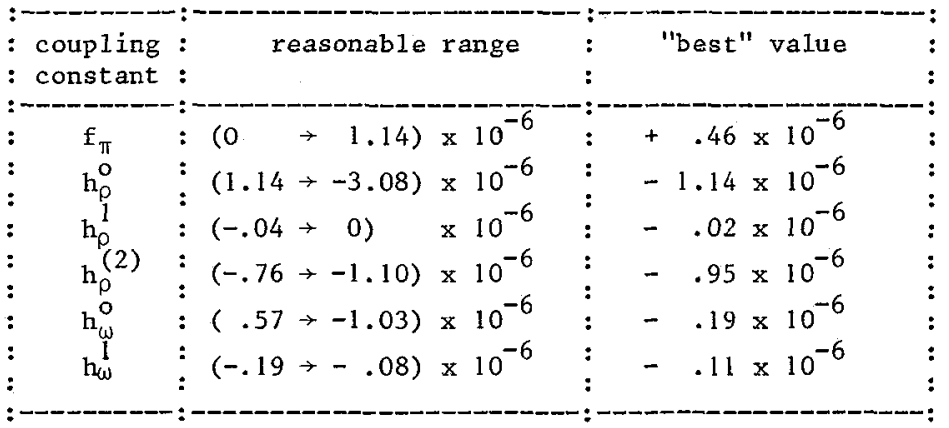

\section{II - FROM THE NN WEAK INTERACTION TO PNC OBSERVABLES}

Two different types of observables have been considered to evidence nuclear paritynon-conservation. A parity forbidden process, such as the well known a decay of $16_{\mathrm{O}}\left(2^{-}, 8.88 \mathrm{MeV}\right)$ which has actually been observed $/ 5 /$, can be looked for. A nonzero correlation between the spin and the momentum of some of the particles involved in a nuclear pracess is a different signature of parity-non-conservation. Most of pnc effects observed so far are of this type. They include a difference in the cross-section for longitudinally-polarized projectiles in proton-nucleus scattering ( $\mathrm{pp}, \mathrm{pd}, \mathrm{p} \alpha, \mathrm{p}{ }^{2} \mathrm{HO}$ ) as well as in thermal neutron-nucleus scattering (n $\mathrm{Br}, \mathrm{n} \mathrm{Sn}, \mathrm{n}$ La...). In some cases, 1ongitudinally polarized ejectiles are considered (circular polarization of $\gamma$ in radiative transitions). They also include an asymmetry in the emission of some final products ( $\gamma$ or fission products) with respect to the polarization of the projectile (neutron in most cases). Finally, the pnc neutron spin rotation in matter, where the reference to a spin-momentum correlation is perhaps less obvious, has been considered. The above list shows that the diversity of processes now studied is very large. Furthermore, the size of the effects goes from the current expected value of $10^{-7}$ to a few $10^{-2}$ in some cases. These features make difficult to treat al1 these processes in detail here and only a few important points, which may cast some light on these differences, are intended to be discussed.

As the force responsible for pne effects is very weak, it is reasonable in all present cases to start with the weak scattering amplitude :

$$
A=\left\langle\psi_{f}^{-}\left|v_{p n c}\right| \psi_{i}^{+}\right\rangle
$$

It involves the matrix element of the pnc interaction between the incoming and outcoming states. For processes involving photons, the amplitude would be

$$
\mathrm{A}_{\gamma}=\left\langle\psi_{\mathrm{f}}^{-}\left|\mathrm{V}_{\mathrm{pnc}} G \mathrm{H}_{\gamma}\right| \psi_{i}^{+}\right\rangle+\left\langle\psi_{f}^{-}\left|\mathrm{H}_{\gamma} G \mathrm{~V}_{\mathrm{pnc}}\right| \psi_{i}^{+}\right\rangle,
$$

where $G$ is the Green's function. Most estimates for observables can be obtained from the above pnc amplitudes, together with the parity conserving ones in some cases. The main problem arises from the description of the incoming (or outcoming) states which have to be eigenstates of the strong interaction. This is particularly important for reaction-type processes where open channels occur.

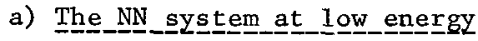

The NN system is the simplest one to describe. Once a model is given for the strong NN interaction, the calculation of the various pnc amplitudes offers no particular difficulty. The only uncertainty arises from the strong interaction at short distances. This is important because most components of the weak potential are short-range ones. There are two aspects to be considered here. The first one 
comes from the short range repulsion whose effects on transitions between $S$ and $P$ NN states due to $\rho$ or $\omega$ exchanges vary by a factor 2 , depending on the strong interaction model. The second one, often neglected in many-body systems, is the effect of the tensor part of the NN interaction whose effect differs from one amplitude to another one. Among the various low energy amplitudes, the $S-P$ transition amplitudes are the most sensitive to the above short-range correlations. As their contributions dominate in low energy nuclear processes, it is appropriate to list them here, together with the corresponding isospin properties of the weak interaction $/ 6 /$ :

$$
\begin{aligned}
& 1 \quad 3 \text { proton-proton ( } \mathrm{pp}): \Delta \mathrm{T}=0,1,2 \\
& \mathrm{~S}_{\mathrm{O}} \leftrightarrow \mathrm{P}_{\mathrm{O}} \quad \text { proton-neutron(pn): } \Delta \mathrm{T}=0,2 \\
& \text { neutron-neutron }(\mathrm{nn}): \Delta \mathrm{T}=0,1,2 \\
& { }^{3} \mathrm{~S}_{1} \leftrightarrow{ }^{1} \mathrm{P}_{1} \quad \text { proton-neutron }(\mathrm{pn}): \Delta \mathrm{T}=0 \\
& { }^{3} \mathrm{~S}_{1} \leftrightarrow{ }^{3} \mathrm{P}_{1} \quad \text { proton-neutron }(\mathrm{pn}): \Delta \mathrm{T}=1
\end{aligned}
$$

Due to their energy dependence, the above amplitudes may appear to carry a large amount of information on the weak potential. In fact, at low energy, this energy dependence is essentially determined by well known features of the strong interaction so that the amplitudes can be parametrized with their threshold values. The same property holds for the NN system within the nuclear medium /7/. As a result, Low energy pnc effects are expected to be determined by a limited number of parameters which may be chosen as the strengths of an effective NN interaction /7/ and would roughly describe the following processes :

$$
\begin{aligned}
& \text { polarized proton } x \text { unpolarized proton } \rightarrow \mathrm{x}_{\mathrm{pp}} \text {, } \\
& \text { polarized proton } x \text { unpolarized neutron } \rightarrow \mathrm{x}_{\mathrm{pn}}^{ \pm} \text {, } \\
& \text { polarized neutron } \mathrm{x} \text { unpolarized proton } \rightarrow \mathrm{x}_{\mathrm{p} n} \text {, } \\
& \text { polarized neutron } x \text { unpolarized neutron } \rightarrow x_{n n} \text {, } \\
& \text { polarized proton } x \text { polarized neutron } \rightarrow \mathrm{x}_{\mathrm{pn}}^{\circ} \text {. }
\end{aligned}
$$

Due to the limited number of parameters which are involved, some consistency of measurements in various low energy processes should be observed.

As far as the NN system is concerned, only experiments in pp scattering have been performed until now and we have therefore to rely on other more complex processes to determine the other four S-P transition amplitudes.

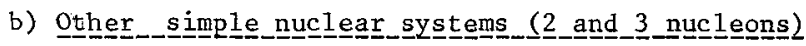

Beside NN scattering, there are processes where a complete theoretical analysis is possible. In the NN system, pnc effects involving photons have been considered, in particular in the thermal neutron radiative capture on protons where experiments intending to improve on previous ones are in progress. Complete theoretical analyses are also possible now in three-nucleon systems by solving the appropriate Faddeev equations. This has been done for nucleon-deuteron scattering /8-10/ and in thermal neutron radiative capture on deuterons, where experiments have been done.

\section{c) Two-_-body_type_syst트는}

Beyond three nucleons, nuclear systems cannot be treated exactly any more. However, to the extent where they can be described as one nucleon moving in the field of a core essentially inert $/ 11 /$, they may be tractable. This approach has underlain most of the studies in the past and is still playing an important role at present. Its validity implies that pnc effects can be wel1 reproduced from an average nucleon-nucleus potential as far as the essential features are concerned. The strengths of the proton and neutron parts of this interaction, $\mathrm{x}_{N}^{\mathrm{P}}$ and $\mathrm{x}_{\mathrm{N}}^{\mathrm{n}}$, can be related to the S-P transition amplitudes, or to the parameters of the weak potential. In our notations, we have : 


$$
\mathrm{x}_{\mathrm{N}}^{\mathrm{P}}=\mathrm{x}_{\mathrm{pp}}+\mathrm{x}_{\mathrm{pn}}^{+}, \mathrm{x}_{\mathrm{N}}^{\mathrm{n}}=\mathrm{x}_{\mathrm{nn}}+\mathrm{x}_{\mathrm{pn}}^{-} \text {, }
$$

The above approach should be appropriate in the case of low energy nucleon bound states and in particular for low energy radiative transitions. Such transitions were extensively studied few years ago and for several of them, there are now both reliable predictions and measurements. The large effect observed in most cases has its origin in the suppression of the regular transition. It is also due to the presence close to the initial or final state of a state with the same angular momentum but opposite parity (parity doublet).

A possible description of pnc effects in a single-particle framework does not completely remove the uncertainties, due to the single-particle wave functions in the case of deformed nuclei for instance $/ 12 /$, and for a long time they have prevented to draw any firm conclusion. In this respect, a major advance has occured since M. Gari's review at a previous workshop /13/. It is due to the relationship which has been established between pnc transitions in $18_{\mathrm{F}}$ and $1{ } \mathrm{~F}$ and first forbidden $\beta^{+}$decays from ${ }^{18} \mathrm{Ne}$ and ${ }^{19} \mathrm{Ne} / 14 /$. It allows to have for the first time an independent check about our previous theoretical estimates /15/ which turned out to be too large by a factor 3 in these nuclei. In the spirit of single-particle transitions considered in this subsection, the suppression factor has its origin in the inclusion of further components (with $\Delta N=2$ ) in the single-particle deformed wave functions and to some pairing-type effect. It is interesting to notice that both effects were considered long ago in some heavy nuclei $/ 16 /$ and it is likely that the possibility of describing pnc effects in ${ }^{19} \mathrm{~F},{ }^{175} \mathrm{Lu}$ and ${ }^{181_{\mathrm{Ta}}}$ with one parameter, $\mathrm{x}_{\mathrm{N}}^{\mathrm{P}}$, is not fortuitous.

As weak interactions do not conserve isospin, the strengths of the proton and neutron-nucleus pnc forces, $\mathrm{X}_{\mathrm{N}}^{\mathrm{P}}$ and $\mathrm{X}_{\mathrm{N}}^{\mathrm{n}}$, need not to be the same and by looking at processes where they appear with dîfferent weights (see Table II), they may be extracted from measurements.

Table II - Expression of some pnc effects in terms of the strengths of the nucleonnucleus interaction

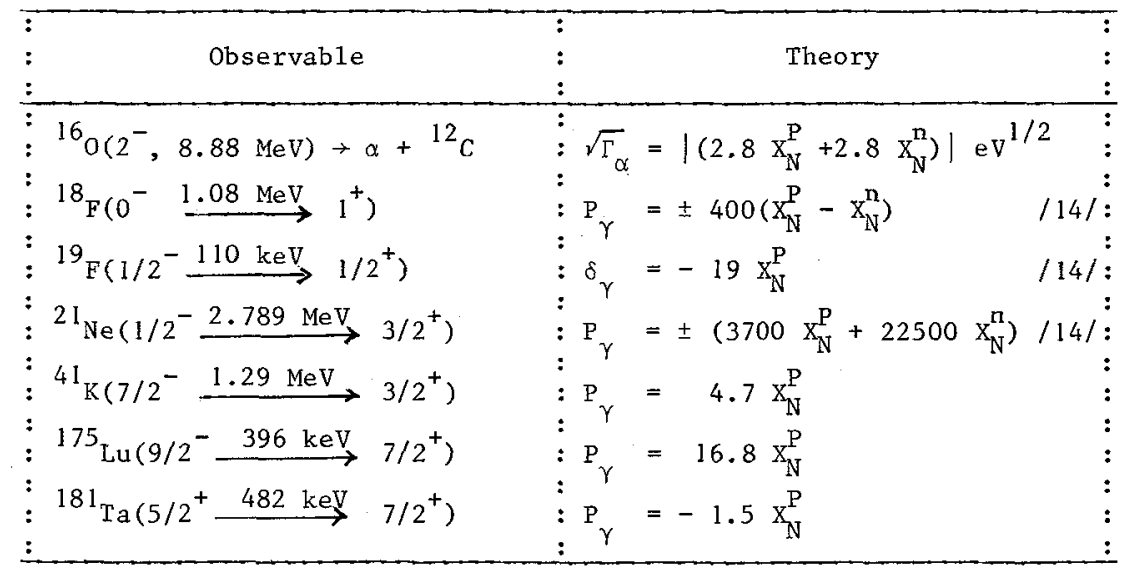

\section{d) More complex nuclear systems}

With an increasing available energy, the coupling to other channels of some system initially in a nucleon-nucleus (g.s.) configuration becomes important. The total state, whose wave function is to be known to calculate the weak pnc amplitudes, eqs 2.3 , can be written as the sum of two different kinds of components : 
$\psi>=\mid$ nucleon-nucleus $(\mathrm{g} \cdot \mathrm{s})>.+\Sigma$ |excited nucleus $>$

The first one represents the entrance channel where the nucleus is assumed to be inert. Its contribution, which can be calculated with different degrees of sophistication, has been retained in several estimates of pnc effects in nucleon-nucleus scattering /17-28/. It offers the advantage of some continuity with what has been learnt about nucleon bound states. The second one has often been neglected on the basis that there is little chance to have a large overlap between such components in the initial and final states. Near a resonance however, the weight of some of these components can be, within the nuclear volume, several orders of magnitude larger than the first nucleon-nucleus component, and thus the corresponding contribution may get strongly enhanced in spite of the fact it is suppressed with other respects /29-33/. As theory is generally unable to describe in detail such components, the relationship between the observables and the weak interaction is here much weaker than when the nucleon-nucleus component is known to dominate. This is likely to be the case in quite different energy ranges : in thermal neutron-nucleus scattering when a resonance is energetically close to the entrance channel, in proton-a scattering at $\mathrm{E}_{\mathrm{Lab}} \approx 50 \mathrm{MeV}$ where $\alpha$-breakup occurs and in proton- ${ }^{2} \mathrm{HO}$ scattering at $\mathrm{P}_{\mathrm{Lab}}=6 \mathrm{GeV} / \mathrm{c}$, where internal excitations of the nucleon are important.

\section{III - COMPARISON BETWEEN THEORY AND EXPERIMENT}

We here compare measurements in several processes to predictions obtained by using the pnc potential given by eq. 1 together with the "best values" of Table I for the meson-nucleon coupling constants. Results for those processes we are going to consider, and which have been extensively studied theoretically and experimentally for most of them, are presented in Table III. Some other processes may have been retained

Table III - Comparison of measurements with predictions made by using the "best" values of Table I for the pnc meson-nucleon coupling constants. References are only mentioned in the case of recent new results. Other references may be found in refs. $34-35$.

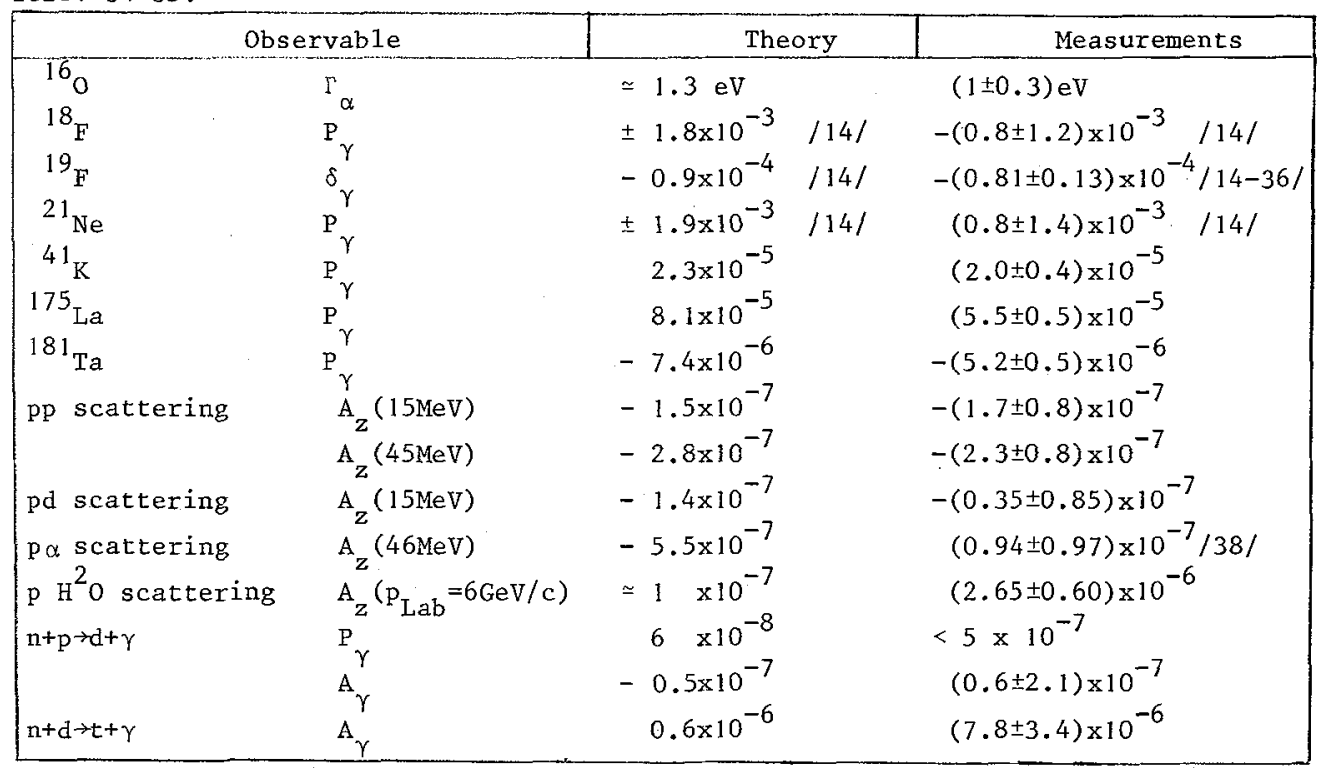

but they should not change the conclusion of the present comparison which is to show that, while theory accounts very well for some measurements, it is not yet completely constrained by them.

Theory and experiment agree quite well in several complex nuclei $\left({ }^{16}, 18_{F} \ldots 181_{\text {Ta }}\right)$, 
in $p p$ and pd scattering and in $n+p \rightarrow d+\gamma$, which may suggest that the potential model given by eq. 1 together with the "best" coupling constants of Table 1 is close to the actual one. There are however noticeable exceptions. Predictions are significantly larger than the measurement in $\mathrm{p}-\alpha$ scattering $/ 18,38 /$ and, at the contrary, much below in $\mathrm{p}^{2}$ HO scattering at very high energy /37/. In both cases, the predictions have been made by neglecting contributions due to the excitation of the target, $\alpha$ or $\mathrm{N}$, which are known to be important. The origin of the present discrepancy may be found in these contributions whose calculations is difficult as mentioned in section 2. It may also be found in the measurements which have been done only by one group until now. In the above discussion, results of ref. /39/ have been discarded. They indeed raise a few questions to which answers have to be given before considering them as a reasonable explanation.

As for demonstrating a theorem, it is not sufficient to emphasize that a theory explains measurements and one has also to show that anyother theory is excluded. This is particularly necessary here because, as evidenced by Table 1 , theory can allow for large deviations with respect to the potential used in making predictions. A careful examination shows that the results in processes where a reasonable agreement is achieved are not completely independent (see Table II) and that sectors of this $\mathrm{NN}$ interaction are better constrained than other ones. One of the best constrained part concerns the pp pnc force whose effects have been observed in longitudinal1y polarized proton-proton scattering at different energies /40/. It would correspond to $X=1.3 \times 10^{-6}$. The second best constrained part concerns the intensity of the profBn-nucleus pnc force which is overdetermined by pnc effects in $19 \mathrm{~F},{ }^{41} \mathrm{~K},{ }^{175} \mathrm{Lu}$ and ${ }^{181} \mathrm{Ta}$. In this determination, ${ }^{19} \mathrm{~F}$ plays a particular role since nuclear uncertainties are better controlled due to information about $\beta^{+}$decay from ${ }^{19} \mathrm{Ne} / 14 /$. Alone, this nucleus would lead to $\mathrm{X}_{\mathrm{N}}^{\mathrm{P}}=4.2 \times 10^{-6}$, whereas with the 3 other odd proton nuclei, it would give a slightly smaller value, $\mathrm{X}_{\mathrm{N}}^{\mathrm{P}}=3.5 \mathrm{x} 10^{-6}$. As the pp pnc force contributes to the proton-nucleus pnc force, it is interesting to see how large is this contribution. Taking into account eq. $4, \mathrm{x}_{\mathrm{N}}^{\mathrm{P}}=\mathrm{X}_{\mathrm{pp}}^{+} \mathrm{x}_{\mathrm{pn}}^{+}$, together with the above values of $x_{N}^{P}$ and $x_{p p}$ leads to the conclusion that the $p p$ pnc force contributes about one-third of the total proton-nucleus pnc force.

Compared to the strength of the proton-nucleus weak force, the strength of the neutron-nucleus force is not so well determined and is constrained to be small with the opposite sign by the absence of sizeabie effect in $21_{\mathrm{Ne}}: \mathrm{x}^{\mathrm{n}}=-\mathrm{x}_{\mathrm{N}}^{\mathrm{P}} / 6 / 41 /$. As any conclusion based on one process is generally known to be Eragile in this field it may be appropriate to delete this nucleus. Then, $\mathrm{X}_{\mathrm{N}}^{\mathrm{n}}$ can take values as large as $\mathrm{X}_{\mathrm{N}}^{\mathrm{p}}$. This contradicts neither results in ${ }^{16} 0$ where theoretical predictions can be easily uncertain by a factor 2 for the decay amplitude, or results in $18_{F}$ where the experimental uncertainty is still large. Pnc interactions involving neutrons are also involved in $\mathrm{n}+\mathrm{p} \rightarrow \mathrm{d}+\gamma$, but the present experimental uncertainty does not allow to draw any firm conclusion. It thus appears that rather little is known with certitude on the pnc interactions of neutrons with nuclei compared to what is known with protons. Further work in this particular sector is desirable.

\section{IV - PARITY-NON-CONSERVATION IN PROCESSES INVOLVING NEUTRONS}

The main source of neutrons for studying pnc nuclear forces is presently provided by reactors. It may be that in a near future, experiments with polarized neutrons of a few MeV be possible in other places, but none of them has been planned until now as far as we know. Furthermore, the usefulness of these experiments involving nuclei with $\mathrm{A}>3$ is perhaps questionable till we understand the origin of the present discrepancy in $\mathrm{p}-\alpha$ scattering $/ 38 /$. If this discrepancy turns out to be due to the nuclear description of the process and in particular to the coupling of the entrance channel to various open channels, then experiments with very low energy neutrons on light nuclei, where the above nuclear effects are less important, will remain for some time the best tool to study pnc neutron-proton and neutronneutron interactions. 
a) Neut ron-proton and neutron-deuteron_scattering

Polarized neutron scattering on protons or neutrons would be the ideal processes to study the above pieces of the pnc NN interaction. The second of these processes can be indirectly studied in neutron-deuteron scattering. Theoretically, there is no difficulty to deal with these processes. As there are no enhancement factor, the effects are likely to be difficult to observe. Nevertheless, some neutron spin rotation in a parahydrogen target, similar to the one observed at ILL in Sn $/ 42 /, \mathrm{Pb} / 43 /$ and La (preprint ILI, B. Heckel et a1.) may be looked for in a near future $/ 44 /$, (B. Hecke1, private communication). Predictions for this rotation amount to $\varphi_{\text {pnc }} / l \simeq 1 \times 10^{-8} \mathrm{rad} / \mathrm{cm}$. A larger effect would indirectly confirm some of the presently unexplained results obtained in other processes and, at the same time, would require some change in the theory,

\section{b) Neutron-proton and_neutron-deuteron radiative capture}

Pnc effects in thermal neutron radiative capture by protons has been considered for a long time both theoretically and experimentally. This process offers several advantages. Its description is rather simple and uncertainties due to mesonic exchange currents, relativistic corrections or a quark phase at short distances, which have been sometimes advocated, turn out to be small when gauge invariance is correctly incorporated. Furthermore, the circular polarization of photons, $P_{\gamma}$, and the asymmetry of their emission with respect to the neutron polarization, $A_{\gamma}$, are respectively sensitive to two different parts of the pnc NN interaction $/ 6 /$; the $\Delta T=0,2$ parts for $P_{\gamma}$ and the $\Delta T=1$ part for $A_{\gamma}$. As there are no particular enhancement of the effects, expectations are quite small. Thus the potential model referred to throughout this paper leads to the following predictions :

$$
P_{\gamma}=0.6 \times 10^{-7}, A_{\gamma}=-0.5 \times 10^{-7}
$$

The most recent measurement of $\mathrm{P}_{\gamma} / 45 /$ has put an upper limit of $5 \times 10^{-7}$, quite consistent with present expectations. Unless measurements presently in progress show a non-zero effect at the level of a few $10^{-7}$, it will be very difficult to reach the accuracy necessary to confirm predictions. The previous measurement of $A_{\gamma} / 46 /$ is also consistent with expectations. Contrary to the experiment on $\mathrm{P}_{\gamma}$, the experiment presently in progress at ILL on $A_{\gamma}$ (M. Avenier, this workshop) should reach an accuracy good enough to support or eliminate some of the potential models proposed in the literature. It will be particularly interesting to compare results of the present experiment to those obtained in ${ }^{18} \mathrm{~F}$, which also depend on the isovector part of the pnc NN forces.

The study of pnc effects in $n+d \rightarrow t+\gamma$ presents features quite different from those evidenced by $n+p \rightarrow d+\gamma$. The normal eross-section is strongly suppressed and therefore some enhancement of the effects is expected. This is partially supported however by the most recent calculations $147 \%$. On the other hand, the various observables (circular polarization of photons, asymmetry in their emission with respect to the neutron or deuteron polarization) are involving all the parts of the NN weak force, in contrast to $\mathrm{n}+\mathrm{p} \rightarrow \mathrm{d}+\gamma$. Uncertainties in theoretical estimates are slightly more important than in $n+p \rightarrow d+\gamma$ and are due for a large part to the unknown ratio of captures in doublet and quartet states. They cannot however explain the large discrepancey between predictions for the asymetry, $A_{\gamma} \simeq 0.6 \times 10^{-6}$ and its measurement $A_{Y}=(7.8 \pm 3.4) \times 10^{-6} / 48 /$. In view of the large error, one has perhaps to wait for a confirmation before seriously worrying with this discrepancy. 


\section{c) Neutron elastic scattering on complex nuclei}

Due to the difficulty to perform exact calculations, the interpretation of pnc effects in systems involving more than 3 nucleons is likely to be uncertain. This is rather unfortunate because there are now very accurate measurements in heavy nuclei and, furthermore, several different pnc effects have been observed for a few of them (Tin in particular). In all these cases, effects are quite large. This is not fortuitous and the enhancement is due to the presence near threshold of a p-wave resonance $/ 21 /$. This is somewhat similar to the enhancement observed in light nuclei involving parity doublets ( $\mathrm{F}$ or $\mathrm{Ne}$ ), but the energy scale is different. A second condition which is often realized is the observation of a large radiative crosssection which is indicative of the existence of a s-wave resonance near threshold.

Two obervables are of special interest at thermal energies : the rotation of the neutron spin in a plane perpendicular to its momentum and the difference in the cross-sections corresponding to different neutron helicities. Assuning that the nucleus has no spin, the pnc forward scattering amplitude may be written as :

$$
f_{\text {pnc }}(0)=G^{\prime}<\vec{\sigma}_{n} \cdot \vec{p}_{n}>\text {. }
$$

In terms of this amplitude, the neutron spin rotation, which has been experimentally studied at ILL for several nuclei, can be written as :

$$
\varphi_{\text {pnc }}=-4 \pi \rho l \operatorname{Re}\left(G^{\prime}\right)
$$

where $p$ is the atomic density of the sample, and $l$ its length. The rotation corresponding to one mean free path, which will be useful in the following, is written as

$$
\varphi_{\text {pnc }}(\text { m.f.p. })=-4 \pi \operatorname{Re}\left(G^{+}\right) / \sigma
$$

where $\sigma$ is the nucleon-nucleus total cross-section. As to the asymmetry in the cross-sections for different neutron helicities, it can be expressed as :

$$
P=\frac{\sigma(+)-\sigma(-)}{\sigma(+)+\sigma(-)}=4 \pi \operatorname{Im}\left(G^{\prime}\right) / \sigma
$$

Both effects involve respectively the real and imaginary parts of the pne amplitude, $f_{\text {pnc }}(0)$, or equivalently $G^{\prime}$. It may seem that $P$ is uninteresting because the numerator, $\sigma(+)-\sigma(-)$, should vanish while the neutron momentum tends to zero. As shown by different authors $129-32 /$, this property does not hold however in the presence of open reaction channels, involving photons for instance.

As we are here concerned by the origin of parity-non-conservation in neutron nucleus scattering, we have to estimate $G^{\prime}$ and try to establish some relationship between this quantity and the weak NN interaction. In a first approximation, it may be assumed that parity-non-conservation in neutron-nucleus interactions is due to the pnc neutron-nucleus force $/ 11,20 /$. As explained in section $2 d$, this is certainly appropriate in absence of possible excitations of the target. Expectations for the spin rotation based on such an approximation are generally quite small $\varphi_{\mathrm{pnc}} / l=1 \times 10^{-8} \mathrm{rad} / \mathrm{cm}$ in $4 \mathrm{He} / 19,26 /, 1 \times 10^{-7} \mathrm{rad} / \mathrm{cm}$ in $\mathrm{Bi} / 22 /$. A larger result has been obtained in ${ }^{40} \mathrm{Ca} / 27 /$, but it is 1 ikely to be reduced by inclusion of finite range effects of the Trexchange pnc NN interaction. Values smaller than the above ones are not excluded if it turns out that the pnc neutron-nucleus force is indeed small as expected from the potential model described in section $I$. The above effects are presently too small to be measurable and it was proposed by M. Forte $/ 21 /$ to look at cases where a p-wave neutron resonance is close to threshold. 
This would result in an enhancement of the effect which may now become measurable.

Estimating pnc effects in the proximity of neutron nucleus resonances is more complicated than in the above "single-particle" approximation since several contributions are possible. Two of them, corresponding to extreme cases, have essentially been considered in the literature. One of them still arises from the neutron-nucleus component. The calculation of this contribution, first retained in earlier estimates $/ 21,20,23,24 /$, has recently been improved by incorporating the true nature of the resonances present at threshold /28/. This has been done by including in the neutron-nucleus-strong interaction potential terms like $\varphi(r) \psi\left(r^{\prime}\right) /\left(E-E_{R}^{\circ}\right)$ representing the effect of coupling the entrance channels to states of the compound nucleus. Results are not so well determined as they used to be. Assuming that such terms are located at the nucleax surface and that the neutron-nucleus interactions are described by square-well type potentials, it can be shown that :

$$
G^{\prime}(s \cdot p \cdot)=-\frac{A}{M^{2}} X_{N}^{n}\left(1-\frac{\sin ^{2}(K R)}{(K R)^{2}}\right) \frac{\left(1+\delta_{s} / p R\right)\left(1+3 \delta_{p} /(p R)^{3}\right)}{\left(\frac{\sin (K R)}{K R}\right)\left(\frac{\sin (K R)}{K R}-\cos (K R)\right.}
$$

Except perhaps for $x_{N}^{n}$, which represents the strength of the neutron-nucleus pnc force, anything is in principle known in this formula. $R$ represents the nuclear radius and $\mathrm{K}$ is related to the depth of the nucleon-nucleus strong force $\left(\mathrm{K}^{2}=2 \mathrm{MV}\right)$. The quantities $\delta_{s}$ and $\delta_{p}$ represent the $s$ and p-wave phase shifts. They can be written as the sum of the potential scattering phase-shift and a term representing the contribution of the nearest resonance :

$$
\delta_{s, p}=\delta_{s, p}^{o}-\Gamma_{s, p}^{n} / 2\left(E-E_{s, p}^{r}+i \Gamma_{s, p}^{\text {tot }} / 2\right) \text {. }
$$

The second contribution which has been considered arises from the parity admixture of states of the compound nucleus. Its expression is given by :

$$
G^{\prime}(c . n .)= \pm \sqrt{\frac{\Gamma_{s}^{n}\left(E_{s}^{r}\right) \Gamma_{p}^{n}\left(E_{p}^{r}\right)}{p_{s}^{r} p_{p}^{r^{3}}}} \frac{|<s| v_{p n c}|p>c \cdot n \cdot|}{\left(E-E_{s}+i \Gamma_{s} / 2\right)\left(E-E_{p}+i \Gamma_{p} / 2\right)}
$$

To estimate this second contribution, one has to precise what is the value of the pnc matrix element between two states of the compound nucleus, $\left\langle s\left|V_{p n c}\right| p\right\rangle c . n$. At first sight, this does not seem easy. Following ideas contained in papers by $R$ : Haas, L.B. Leipuner and R.K. Adair /49/ or R.J. Blin-Stoyle /50/, it is however possible to provide some order of magnitude :

$$
\left.|<s| \nabla_{p n c}|p\rangle_{c . n .}|=|<v_{p n c}\right\rangle_{\text {s.p. }} \mid N^{-1 / 2}
$$

where $\left\langle V_{\text {pnc }}\right\rangle_{s . p .}$ is a typical single-particle matrix element and $N$ the number of configurations appearing in the expansion of the states of the compound nucleus, $\mid s>$ or $\mid p>$. Roughly, this number can be taken as the number of states of the compound nucleus within $1 \mathrm{MeV}$. It is inversely proportional to their average energy spacing and can vary from $10^{4}$ in nuclei with $A \cong 100$ to $10^{6}$ in nuclei with $A \simeq 240$. Information on pnc effects in odd-proton nuclei provide an estimate for $\left\langle V_{p n c}\right\rangle s . p$. From the one observed in $19 \mathrm{~F} / 14 /$, one gets $\mid<V_{\text {pnc }}>$ s.p. $\mid=0.5 \mathrm{eV}$, so that 


$$
|<s| \mathrm{v}_{\mathrm{pnc}}|\mathrm{p}\rangle_{\mathrm{c} . \mathrm{n} !} \mid \simeq 0.5 \mathrm{eV} \times\left(10^{-2}-10^{-3}\right)
$$

To emphasize the statistical character of the above estimate, and also because we are interested here in understanding all the mechanisms which lead from the pnc interaction at the quark level to pnc effects in complex nuclei, we will briefly sketch the details of the demonstration. It can be first shown that each matrix element can be written as a sum of $\mathrm{N}^{2}$ terms whose $\mathrm{N}$ only have a non-zero value. This value is equal to $\left\langle\mathrm{V}_{\text {pnc }}>\mathrm{s} . \mathrm{N}\right.$ on the average, but its sign is underdetermined. One then uses the fact that ${ }^{p_{\text {the }}}$ sum of $\mathrm{N}$ terms equal to $\left\langle\mathrm{V}_{\mathrm{pnc}}{ }^{\mathrm{s}} \mathrm{s} . \mathrm{p}\right.$. IN with signs at random is equal on a quadratic average to $\pm\left\langle\mathrm{V}_{\mathrm{pnc}} \mathrm{s} \cdot \mathrm{p}.\right\rangle / \mathrm{N} 1 / 2$.

We can now compare the two contributions $G^{\prime}(s . p$.$) and G^{\prime}(c . n$.$) . The first$ contribution is in principle well determined in magnitude and sign, which is particularly the case for ${ }^{139} \mathrm{La}$. There may be however cases where the denominator in $G^{\prime}$ (s.p.) is quite small, thus giving rise to some enhancement, but with a sign not well determined (this is perhaps the case in $117_{\mathrm{Sn}}$ ). The second contribution, $G^{\prime}(c . n$.$) , is much less determined in magnitude as explained above, and its sign is$ at random. Both contributions are enhanced by $s$ and $p$-wave resonances near threshold as evidenced by the presence of factors $1 /\left(E-E_{S}+i \Gamma_{S} / 2\right)$ and $1 /\left(E-E_{p}+i \Gamma_{p} / 2\right)$. When such conditions are realized, one expects the following relation between the spin rotation and the asymetry in the cross-section :

$$
\frac{P}{\varphi \text { (m.f.p.) }}=-\frac{\operatorname{Im}\left(G^{\prime}\right)}{\operatorname{Re}\left(G^{\prime}\right)}=\frac{P_{p}\left(E-E_{s}\right)+\Gamma_{s}\left(E-E_{p}\right)}{\left.2\left[\left(E-E_{s}\right)\left(E-E_{p}\right)-\Gamma_{s} \Gamma_{p} / 4\right)\right]}
$$

One also expects some relation between the asymmetry in the cross-section at the $\mathrm{p}$-wave resonance position and at thermal energy. The relation which is actually considered is

$$
P(\text { res. }) / P_{t h}=\sigma_{t h} / \sigma_{p} \times\left(2 E_{p} / \Gamma_{p}\right)^{2},
$$

where $\sigma_{p}$ is the contribution to the cross-section due to the p-wave resonance at its maximum.

In comparing theory and experiment, two aspects are to be considered, the validity of the above relations, eqs. $(8,9)$, and the magnitude of the effects. It appears that these relations are quite well verified when a comparison is possible. As this comparison is 1 ikely to be done at the workshop by the authors of the experiments $/ 43$, $51,52 /$ we will not expand on it here. The good agreement tells us that the nuclear mechanism which provides a large enhancement of the effects is essential1y correct. The magnitude of the effects, which is our main concern in this review, is reproduced with values of $\mid\left\langle s\left|v_{\text {pnc }}\right| \mathrm{p}\right\rangle \mathrm{c} . \mathrm{n}$. $\mid$ which vary from $0.4 \times 10^{-3} \mathrm{eV}$ to $3 \times 10^{-3} \mathrm{eV}$ for nuclei with $A \simeq 100 / 52 \%$. This is quite consistent with estimates given by eq. 7 . However, a study in 139 La shows that the pnc spin rotation observed in this nucleus could be reproduced as well with the contribution of the neutron-nucleus component provided that the strength of the neutron-nucleus pnc force is of the order of $\mathrm{X}_{\mathrm{N}}^{\mathrm{n}}=-4 \times 10^{-6}$. This value is comparable to the strength of the proton-nucleus pnc force, $x_{N}^{p}=3.5 \times 10^{-6}$, mentioned in section 3 . The fact that the contribution from the compound nucleus be as large as the contribution of the neutron-nucleus component in $139 \mathrm{La}$ is consistent with the assertion made in ref. /30/ that the first contribution should dominate over the other one when the average energy spacing of the states of the compound nucleus becomes smaller than $\simeq 100 \mathrm{eV}$ (The average spacing is of about $200 \mathrm{eV}$ in La). Thus, the precise origin of pnc effects may be somewhat obscured in some cases and studies such as those proposed in ref. /33/ should probably be done for determining which picture is the correct one. 


\section{d) Neutron inelastic scattering on complex nuclei.}

We will not consider here pnc effects in longitudinally polarized neutron inelastic scattering which are the counter-part of those already discussed in elastic scattering. The pnc effects of interest here are the asymetries in the emission of some particles, light fission products or photons, with respect to the neutron polarization. These effects were observed long ago for individual radiative channels where their size is not unexpected when the enhancement mechanism described in the previous subsection is present. Pnc effects observed in inclusive reactions /53,54/ are more surprising. One indeed expects that signs for individual channels be at random and therefore the integrated effect should be quite small.

The estimate of pnc effects in inelastic scattering is much more complicated than in elastic scattering. In electromagnetic processes, a complete knowledge of the wave function, including both the parity-conserving and the parity non-conserving parts (eq. 3) is required, whereas only knowledge on the first one is necessary in elastic scattering (eq. 2). This difficulty is perhaps at the origin of the absence of explanation for those effects which have been observed in "inclusive" radiative capture on $\mathrm{Cl}, \mathrm{Br}, \mathrm{Sn}$ and $\mathrm{La} / 54 /$. An attempt in La based on the direct capture process, which is known to work in some neighbouring nuclei gives an effect one order of magnitude larger than the observed one, but completely faills to reproduce the radiative cross-section (work in collaboration with $S$. Noguera). It may be that the mechanism which works in producing a sizeable matrix element $\left\langle s\left|V_{p n c}\right| p\right\rangle c . n$. works again here. This has been shown in a very schematic model. If it is shown to work in actual cases, this explanation would have the great advantage to avoid to require further hypothesis about nuclear structure effects. Arguments which have been proposed to explain the pnc effects observed in fission have been somewhat discussed in the literature $/ 29,30 /$. We will not judge here of their validity and just will note that the hypothesis of same relative phases for fission amplitudes from $s$ and p-wave capture states to various channels, also explains the parity conserving left-right asymetry of light fission products following capture of polarized neutrons $/ 30 /$.

\section{Conclusion}

Understanding the origin of pnc neutron-nucleus interactions implies that we have some control on the different subnuclear or nuclear structure effects which lead from the pnc quark-quark interaction to the pnc NN interaction, and from this interaction to pnc observables in complex nuclei. In deriving the pnc NN interaction,knowledge about the strong NN interaction or about hyperon decays is used. This information however, does not completely determine the strength of the different parts of the pnc NN force and, to make predictions, one is forced to make choices, as reasonable as possible, about unknown inputs. Conversely, the study of pnc effects should tell us what are the correct choices. To calculate pnc effects in nuclear systems, a good description of nuclei under consideration is necessary. It is now available in systems with 2 or 3 nucleons and perhaps a few complex nuclei where it is possible to check independently the wave function. Pnc effects observed in these systems are in quite good agreement with predictions, without requiring extreme hypothesis. In some cases, the agreement is not trivia1. A careful examination shows however that checking completely the potential model used in these predictions would require further experiments, with polarized neutrons in particular.

Two kinds of experiments involving neutrons may be considered. There are experiments in simple systems, $n+p, n+d$, where pnc effects are expected to be difficult to measure, but which offer the advantage of an unambiguous interpretation. The example of the $\gamma$ asymmetry in $n+p \rightarrow d+\gamma$ shows that such experiments are not inacessible and should be encouraged. There are experiments in complex nuclei where the presence of a $\mathrm{p}$-wave resonance near threshold in particular favors the observation of pnc effects which have indeed been observed in several of them. In elastic scattering, the different experiments performed on the same nucleus establish without any doubt the important rôle played by these resonances. The magnitude of the effect is consistent with 
the broad range expected for them in the case where the effect is due to the parity admixture of states of the compound nucleus. Due to the complexity of nuclear structure effects, the information to get from them on the pure NN interaction is rather limited. They will have however evidenced the rôle of some nuclear structure effects, such as the opening of reaction channels, which have often been neglected and might contain the key necessary to understand parity non-conservation in simpler systems such as $\mathrm{p}-\alpha$ scattering. Several of these experiments have been performed with the hope to get some reliable information on the neutron-mucleus force. It is not clear whether this force plays an important rôle in effects presently observed in heavy nuclei, but one cannot exclude it will do it in some nuclei which have still to be detected. Finally the pnc effects observed in inclusive reactions, fission or radiative capture, are perhaps the only ones where a reasonable explanation is lacking. The sensitivity to the nuclear description reachesits maximum in these processes and it is conceivable, as mentioned by different authors, that parity non-conservation, instead of being the object of the study, is becoming a tool to learn about very subtle features of nuclear dynamics.

Among people involved in preparing this report, we would particularly like to thank $\mathrm{Dr}$. S. Noguera for his collaboration in some of the results presented here as well as Drs.B. Heckel and M. Avenier for providing us with information concerning their experiments.

\section{Ref erences}

/1/ GLASHOW, S.L., Nuc1. Phys. 22 (1961) 579. WEINBERG S., Phys. Rev. Lett. 19 (1967) 1264, 27 (1971) 1688.

SALAM A., in "Elementary Particle Theory : Relativistic Groups and Analiticity" N. Svartholm ed., p. 367, Almqvist and Wikse11, Stockholm, 1968.

12/ BARTON G., Nuovo Cimento 19 (1961) 512.

13/ DESPLANQUES B., DONOGHUE J.F. and HOLSTEIN B.R., Ann. of Phys. 124 (1980) 449.

$14 /$ HÖHLER C. and PIETARINEN E., Nuc1. Phys. B95 (1975) 210.

15/ NEUBECK K., SCHOBER H. and WÄFFLER H., Phys. Rev. ClO (1974) 320.

$16 /$ DANILOV G.S., Phys. Lett. 18 (1965) 40.

$17 /$ DESPLANQUES B, and MISSIMER J., Nucl. Phys. A300 (1978) 286.

18/ DESPlanQUES B., BENAYOUN J.J. and GIGNOUX C., Nucl. Phys. A324 (1979) 221.

/9/ KLOET W.M. et al.,Phys. Rev. C. 27 (1983) 2529.

/10/ AVISHAI Y., Nuc1. Phys. A399 (1983) 575.

/11/ MICHEL C., Phys. Rev., $1 \overline{33}$ (1963) B329.

/12/ DESPlanQues B., Nuc1. Phys. A316 (1979) 244.

/13/ GARI M., Inst. Phys. Conf. Ser. No 42 (1978) 70.

114/ ADELBERGER E.G. et al., Phys. Rev. C2.7 (1983) 2833.

/15/ GARI M., McGRORY J.B., and OFFERMANN F., Phys. Lett. 55B (1975) 277. BRANDENBURG R.A., MCKELLAR B.H.J. and MORRISON I., Phys: Rev. Lett. 41 (1978) 618 .

BINI M., BIZZETI P.G. and SONA P., Phys. Rev. C23 (1981) 1265. HAXTON W.C., GIBSON B.F. and HENLEY E.M., Phys. Rev. Lett. 45 (1980) 1677.

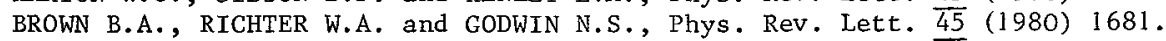

116/ DESPLANQUeS B, and VINH MAU N., Phys, Lett. 35B (1971) 28.

/17/ HENLEY E.M. and WOLFENSTEIN L., Nuc1. Phys. A300 (1978) 265. HENLEY E.M., $\quad$ Nucl. Phys. A $\overline{300}$ (1978) 273.

/18/ ROSER Th. and SIMONIUS M., Polarization Phenomena in Nuclear Physics, 1980, AIP Conference Proceedings $n^{\circ} 69$, ed. G.G. O1sen, p. 1446.

$/ 19 /$ DMITRIEV V.F. et al. Phys. Lett. 125 (1983).1.

/20/ STODOLSKY L., Phys. Rev. 50B (1974) 352.

$121 /$ FORTE M., Inst. Phys. Conf. Ser. $\mathrm{N}^{\circ} 42$ (1978) 80.

/22/ TADIC D. and BARROSO A., Nucl. Phys. A294 (1978) 376.

/23/ BaRRoso A., and MARGACA F.M., J. Phys. G., Nuc1. Phys. 6 (1980) 657.

/24/ KARL G. and TADIC D., Phys. Rev. C20 (1979) 1959.

125/ BERNABEU J. and NOGUERA S., Nucl. Phys., ed. C.H. Dasso, Noth-Holland, Amsterdam, 1982, p. 755.

126/ AVISHAI Y., Phys. Lett. 112B (1982) 311. 
127/ AVISHAI Y., Phys. Rev. C 28 (1983) 656.

$128 /$ DESPLANQUES B. and NOGUERA S., Proceedings of the International Conference on Nuclear Physics, Florence 1983, Vol. 1.

/29/ SUSHKOV 0.P. and FLAMBAUM V.V., Sov. Phys. Usp. 25 (1982) 1.

/30/ BUNAKOV V.E. and GUDKOV V.P., Nucl. Phys. A401 (1983) 93.

$131 /$ LOBOV G.A., Sov. J. Nuc1. Phys. 35 (1982) $8 \overline{22 .}$

132/ STODOLSKY L., Nuc1. Phys. B 197 (1982) 213.

133/ NOGUERA S. and BERNABEU J., Phys. Lett. 129 (1983) 125.

$134 /$ DESPlanques B., Nuc1. Phys. A 335 (1980) 147.

135/ DESPLANQUES B., Proceedings of the 8 th Workshop on Weak Interactions and Neutrinos, JAVEA, 1982.

136/ ELSENER K. et al., Proceedings of the International Conference on Nuclear Physics, Florence, 1983, Vo1. 1.

137/ BARROSO A., Proceedings of the Workshop on Weak Interactions and Neutrinos, JAVEA, 1982 .

133/ HENNECK R. et al., Phys. Rev. Lett. 48 (1982) 725 and Proceedings of the International Conference on Nuclear Physics, Florence, 1983, Vo1. 1.

139/ NARDULLI G. and PREPARATA G., Phys. Lett. 117B (1982) 445.

140/ POTTER J.M. et a1., High Energy Physics with Polarized Beams and Polarized Targets, ed. G.H. Thomas, AIP Conference Proc. 51 (1978).

HENNECK R., et al., Phys. Rev. lett. 44 (1980) 699 and Polarization Phenomena, 1980 ed. G.G. Ohlsen, AIP Conference Proc. 69 (1981).

VON ROSSEN P., VON ROSSEN U. and CONZETT H.E., same ref. as above.

TANNER D.M. et al., Proceedings of the International Conference on Nuclear Physics, Florence, 1983, Vol. 1 .

141/ SNOVER K.A. et al., Phys. Rev. Lett. 41 (1978) 145 and Ref. 14.

/42/ FORTE M. et al. Phys. Rev. Lett. 45 (1 $\overline{980}$ ) 2088.

$143 /$ HECKEL B. et a1., Phys. Lett. $11 \overline{9 B}$ (1982) 298.

144/ SEREBROV A.P., Proceedings of the XIV Winter School of the L.I.Ya.F., for Nucl. and Elem. Part. Phys. USSR Acad. Sci. 28 (1979).

145/ V.M. LOBASHEV, Proceedings of the $8 \mathrm{th}$ Workshop on Weak Interactions and Neutrinos, JAVEA, 1982.

146/ CAVAIGNAC J.F., VIGNON B. and WILSON R., Phys. Lett. 67B (1977) 148.

$147 /$ DESPLANQUES B. and BENAYOUN J.J., Proceedings of the Ninth International Conference on the Few Body Problem, Eugene 1980, ed. F.S. Levin.

148/ AVENIER M., Thesis, University of Grenoble (1982).

149/ HAAS R., LEIPUNER L.B. and ADAIR R.K., Phys. Rev. 116 (1959) 1221.

/50/ BLIN-STOYLE R.J., Phys. Rev. 120 (1960) 181.

$151 /$ KOLOMENSKY E.A. et al., Phys. Lett. 107B (1981) 272.

$152 /$ ALFIMENKOV V.P. et al., Nuc1. Phys, A398 (1983) 93.

153/ DANILYAN G.V. et al., Proceedings of the International Conference on Nuclear Physics, Berkeley, 1980, Abstracts p. 371. VESNA V.A. et a1. JETP Lett. 31 (1980) 663. ANDREEV V.N. et a1., JETP Lett. 31 (1980) 530 , PETUKHOV A.K. et a1., JETP Lett. 32 (1980) 300.

154/ VESNA V.A. et al., JETP Lett. 36 (1982) 209. 